\title{
Shmuel Zaks - The Early Years: \\ A Combinatorialist in Distributed Computing
}

\author{
Nicola Santoro \\ School of Computer Science, Carleton University, Ottawa, K1S 5B6, Canada
}

\begin{abstract}
Celebrating Shmuels Zaks' 60th birthday and his remarkable career, the focus of this talk is on his early contributions to Distributed Computing. In particular, in this talk I examine how this young combinatorialist/graph theorist, upon discovering the beauty and fun of distributed algorithms, was so captured by the area that he never left it. In these early explorations, his research contributions have been many, some very important (e.g. lower bound for election in complete graphs) and some very beautiful (e.g. guessing games in synchronous networks). In this talk, a few of these research results are described and commented, and some of his other contributions to the Distributed Computing community during those years are highlighted.
\end{abstract}

